endocrine examinations such as urinary steroides, BMR, Thorn's test, serum cholesterol, serum protein analysis and serum electrolytes.

5) Psychological test and life history were inquired carefully and precisely. Only 3 cases showed normal profile in MMPI, 12 cases were the 1, 3, type which displays a hypochondrical-hysterical state.

From the Kraepelin test only 3 cases showed a normal curve, while the others showed a lack of initial endeavour or recess effect. Interview of their life history is essential in arriving to the conclusion. Past history of psychosomatic disease (18 cases), death of their parent in childhood (17), changing of job (18), changing of doctors for the injury (21), compensation not settled (21) are the most common causes. Almost all of these patients were the injured (18), and they either hate or are angry with the assaulter.

From these investigation we can indicate that there is an intimate relation with adaptation disorders in each cases. It is important to deal with these intractable cases from the standpoint of holistic aspect.

\title{
182. Studies on So-called Whiplash Injury
}

\author{
Yoshihiko Watanabe and Fumio Karube \\ Department of Orthopedic Surgery and Neurosurgery, Shimizu Kosei Hosiptal \\ Koji SHIbUYA \\ Department of Orthopedic Surgery, School of Medicine, University of Chiba
}

199 cases, suffered from extension acceleration injury in the neck by rear end collision, were treated and followed up. The cases who had the complication of the injury in the spine, the spinal cord, and the brain were removed from this report.

Eelectromyography was achieved on 33 cases with at random selection. On 29 cases, abnormal waves such as high amplitude voltage, complex neuro-muscular unit voltage, grouping voltage and so on were noticed. The relationship among electromyogram, and their symptoms was not definite. Re-investigation on 8 cases was shown that abnormal electromyogram gradually appeared in the upper part of the cervical cord day by day. This inclination was not parallel with ohter symptoms.

Electroencephalography was achieved on 34 cases with at random selection. 4 cases were abnormal and 5 cases borderline. But, on the cases of normal electroencephalogram, the appearance of alpha-waves was apt to extend diffusely. The relationship between electroencephalogram and symptoms was not proven directly, but the data of electroencephalogram was useful for the administration of drug 
(anticonvulsant was sometimes effective on the complaints on the neck an shoulder). That is, it might be speculated that some sympoms are embellishe with the brain-disorders.

Obstinate cases in the chronic stage were able to classify to two group: that is, one group ( 7 cases) had chiefly the complaints of headache, migraine an neck occipital symptoms, and another group ( 6 cases) had pain in the neck an shoulder girdle. They said that first group was derived from the disorders $i$ the vertebral artery, vertebral nerves, and cervical sympathetic nerves. But, o our experiences, patient's complaints re-occurred on the discography at the uppe cervical spine (C2-3, C3-4). As a brace without loading and anterior spini fusion were effective, the intervertebral disc lesions in the upper cervical spin were thought for primary cause of this disease. On second group, traction, brace without loading, scalenotomy, and anterior spinal fusion were effective.

Based on these findings, the following conclusions were drawn that an ob stinate case of whiplash injury must be treated as an intervertebral disc lesion a the cervical spine.

\title{
183. About So-Called "Whiplash Injury" in Neurosurgical Field
}

\author{
Hiroki HiraI, Masayuki Takeda, Shuji OhKawara and Teruyoshi Hashiba \\ Department of Neurosurgery, Sapporo Medical College
}

We have experienced 2967 cases of trauma to the head and/or the necl due to traffic accident during past 2 years and 5 months in the Sapporo Medica College Hospital.

This report reveals the analystic data about the cases of so-called whiplas: injury only.

As to sex distribution, male was $69.6 \%$, female $30.4 \%$. $58.2 \%$ of case was caused by rear-end collision. The disturbance of consciousness was observer in $53.7 \%$ of cases, $66 \%$ of cases began to complain of more or less certain symp toms within 3 days. In the X-ray examination, $13.9 \%$ of cases reveals sligh compressed fracture, $19.5 \%$ of cases cervical instability, $10.2 \%$ of cases narrow ing of intervertebral space and $54.1 \%$ no changes.

In 2 cases with typical great occipito-trigeminal syndrome in which Cloward procedure was carried out for the lower cervical part, the headache in the occipt was markedly improved. From this results we are impressed that there might $b$ some unknown mechanism which results in great occipito-trigeminal syndrom not only in the upper cervical part, but even in the lower cervical part.

As to the prognosis, in $26.4 \%$ of cases the sign and symptom were subside 